\title{
BEE INSPIRED AGENT BASED ROUTING PROTOCOL-SECONDARY USER (BIABRP-SU)
}

\author{
Ramesh Palanisamy \\ Research Scholar,AMET University, Chennai \\ rameshphd26@gmail.com \\ V. Mathivanan \\ Research Supervisor,AMET University, Chennai \\ vmathivanan@yahoo.com
}

\begin{abstract}
Design and development of routing protocol is one among the plunge research in cognitive radio mobile ad hoc networks (CRAHNs). Swarm intelligence is a fairly new-fangled loom to problem solving so as to acquire inspiration from the social behaviors of insects, such as ants and bees. Self-organization, decentralization, adaptivity, robustness, and scalability make swarm intelligence a successful devise archetype for routing in CRAHNs. This third contribution to the doctoral research aims in design and development of bee inspired agent based routing protocol (BIABRP) for CRAHNs. Adaptive scouting, adaptive foraging, optimal path selection and detection of path removals are performed using honey bee inspired strategy. NS2 simulation tool is used to examine the performance of the proposed BIABRP. Appropriate performance metrics are chosen in order to conduct performance evaluation and the results prove that the proposed BIABRP outperforms in terms of packet delivery ratio, end-to-end latency and primary users activity.
\end{abstract}

\section{Keywords:}

CRAHN, Cognitive, BIABRP, Bee, Routing.

\section{INTRODUCTION}

Cognitive radio (CR) is an attracting progression that allows cognitive clients to take a shot at the void parts of the range assigned to essential clients. CR is completely considered as a promising advancement that approaches with the range insufficiency issue fulfilled by the present inflexible range part strategy. It is readied for perceiving its radio surroundings and adaptively recognizing so as to pick transmission parameters as demonstrated results, which upgrades CR structure execution and abstains from bursting in with Discharge. Late research activities drove in CR have basically centered around clever range access and range use. Regardless, CR change will have colossal impact on upper layer execution in remote networks, particularly in adaptable strikingly doled out networks (MANETs), which attract remote contraptions to constantly make networks without on an exceptionally vital level using a settled base. Particularly, issues in non-cognitive MANETs all things considered still of readiness for the CR perspective. Regardless, some unmistakable characteristics of CRs adjust new nontrivial issues with CR-MANETs. Yet a couple tries have been done to the medium access control (Macintosh) layer issues, routing is still one of the particularly key networking issues in CR-MANETs. From a routing perspective, it is common that information packs are composed by framework for a continuing and strong approach to manage keep away from unending rerouting issues coming to fruition to dynamic rerouting may instigate show tempests to the system, manhandle the unprecedented radio resources, and savage end-to-end system execution, for instance, throughput and deferral. Isolated and standard MANETs, a path in CRMANETs is particularly flimsy since it is impacted by the movability of CUs and moreover by the deterrent to Discharge as well. There are an extensive number of routing protocols proposed in established MANETs, for example, destination-sequenced distance vector routing, dynamic source routing (DSR), and ad hoc on-demand distance vector (AODV) routing. Then again, it is hard to apply them straightforwardly to CR-MANETs because of the particular qualities depicted previously. On the other hand, it may not be attractive to outline another routing protocol devoted to CR-MANETs because of the development and accessibility of existing routing protocols. 


\section{LITERATURE REVIEW}

Janacik, P et al.,20.13 provided a broad overview of ant colony optimization-based routing protocols, while focusing on four selected approaches it described the operation and discussing their properties in detail for ad hoc networks. Kiran, K et al.,2014 described the implementation of a framework in which a multi-hop, adhoc network is deployed with hybrid nodes to enhance network throughput. Routing algorithm implemented was be e-hive algorithm. Karthikeyan, D and Dharmalingam, M.,2013 proposed an energy efficient routing algorithm for MANETs based on Ant Colony Optimization for minimizing energy consumption of the nodes and prolong the life of the overall communication system. In Desai, R.and Patil, B.P.,2015, agents were used to optimize the performance of a network on trial and error basis. It also described the algorithm used in cooperative reinforcement learning approach. Prasad, B.M.G et al.,2013 aimed to perform the load balancing and congestion endurance in ad hoc network. Zafar, S.; Soni, M.K.,2014 developed a prospective approach which was a trust based QOS protocol (TBQP) using meta-heuristic genetic algorithm for optimizing and securing MANET. Genetic algorithm will help in maintaining Quality of Service(QOS) by selecting a fittest. Kuen-Han Li et al.,2013 proposed Ant-Based On-Demand Clustering Routing Protocol which uses ant-like agents inspired by the foraging behavior of ant colonies to explore networks and discover a route which fulfills several QoS requirements including minimal traffic overhead or energy-efficiency for MANET. Sharma, P.; Kumar, R.,2014 proposed a scheduling algorithm based on weights is proposed. It aimed to calculate the optimized path from source to destination for sending data, where it used swarm intelligence to find the optimal path for flow of data and information. Chengetanai et al.,2015 presented an overview of swarm intelligence routing techniques that are applicable to MANETs. It investigated the methods of swarm intelligence algorithms which in turn can be used to effectively route data packets on a wireless MANET. Thangadurai, $\mathrm{K}$ and Anchugam, C.V.,2014 proposed a approach called fuzzy ant colony based routing protocol using fuzzy logic and SI of different objectives while retaining the merits of swarm based intelligence algorithm for ad hoc networks.

\section{BEE INSPIRED AGENT BASED ROUTING PROTOCOL (BIABRP)}

BIABRP is another honeybee moved flexible routing protocol in light of the communitarian practices of honeybee foragers. Taking after a reactive technique, BIABRP's honeybee agents research the topology exactly when data are required to be transmitted between nodes. They are proposed to screen and evaluate the discovered execution ways and select the perfect one in perspective of a decision instrument charged by their normal accomplices. Excited by Swarm Learning, the proposed protocol makes different suspicions as for the agents being utilized, their behavior in conclusion the correspondence between them. These suspicions are planned to blueprint contrasting thoughts of their trademark accomplices. Beside the obvious mapping; a source node is seen as the honeybee hive, the destination is the sprout or wellspring of support, and the transitional nodes the way or flying detachment to be crossed from the hive to the bloom, in BIABRP.

Like nature, an upgraded way triggers more agents to take a shot at it by using a honeybee's comparability enlistment. Change after some time offers an adaptivity which not simply is a trademark that can be mapped from nature to frameworks; it is also one of the essential contemplations behind any flexible routing protocol in the field of SI-based frameworks organization. Conforming to have the ability to respond to changes routing, not simply updates the self-repairing piece of the protocol, it moreover helps its flexibility to unmistakable framework conditions and sizes.

BIABRP's agents have the ability to adaptively work in parallel, talk with each other to exchange direct information that is purposely assembled from the framework and achieve multi-path routing at an overall level. Losing a pros (pack incident) or even a center node of a way is clearly an aggravation to a proposed routing plan. Before long, BIABRP's SI parts allow the protocol to feasibly handle dissatisfactions and invoke elective courses of action or acquaint new scouting methodology with find some new. Scattering is proficient and in this way, the computational costs are spread over the nodes that take an enthusiasm to each particular framework development. The most crucial internal frameworks of the protocol are inspected in the accompanying range. In particular, the adaptable scouting, scavenging, perfect way decision and acknowledgment of way disillusionments.

\subsection{Adaptive Scouting}

Exactly when a course is required at the source node and no past information is open, a scout pack is made and sent by show to whatever is left of the framework. The scout bundle is responsible for finding open approaches to its destination, while it familiarizes neighboring nodes with each other, in a ricochet by hop way. In case the getting node is the needed destination, an ack_scout pack is made and sent back to the source to attest a viable way. Unicast is used instead of appear, in light of the way that the ack_scout most likely knows which nodes to visit remembering the deciding objective to give back, that is, the route's inverse the scout explored regardless. A destination node is allowed to make various ack_scouts, dependent upon the amount of scouts it gets from the framework, allowing different approaches to be found. Moreover, the destination node 
doles out to every discovered way a stand-out recognizing confirmation number. In the midst of the enterprise back, ack_scouts dependably accumulate and pass on low-level data got from the lower layers, starting with one node then onto the next.

Data transmission is proficient by making, stacking and sending forager groups. Each forager is accountable for passing on data payload got from the vehicle layer. At the source node, the decision instrument for the most suitable route depends on upon the fancied behavior of the protocol, subject to its utilization. The default determination relies on upon the speediest route between the source and the destination. At each most of the way node, the accompanying bob is easily recouped from the area routing information, in light of the forager's bearing and the conspicuous verification number that it passes on. This approach is impelled by the source routing $[24,53]$ met in other reactive protocols, for instance, DSR. Regardless, in BIABRP the forager group does not need to pass on the whole path within its header. Maybe, every transitional node most likely knows which ways it is a person from, and considering the forager's heading, it can pick the accompanying node towards the destination's wellspring. At the destination node, the forager package transforms into an ack forager package and stays in a pad line until it is again alloted to pass on data back to the source. This behavior duplicates the veritable honeybees which keep centered bloom for a long time assembling the required thing, for instance, dust or nectar. For booking purposes they are utilized as a part of a first-in-first-out configuration. The accompanying ricochet determination for an ack_forager is then done again by considering the forager's heading.

Searching in nature involves a couple stages; a honeybee being enrolled to take after a specific mission, heading out to the target wellspring of food, assembling the genuine dust, nectar or water and passing on it back to the hive. Exactly when a honeybee completes a looking cycle, thusly she is back at the hive, she can either perform a honeybee move to share best in class information about the scavenging, or ignore it and focus her thought on something else. The decision relies on upon a quality's percentage components, for instance, the sweetness of sugar, the faultlessness of taste, the sum and effortlessness of getting, the detachment, the blossom's sort, et cetera.

BIABRP foragers are planned to take after practically identical norms. In particular, ack_foragers have the ability to judge the way's temperament and recognize any possible change or crumbling after some time. Checking a way's quality is both a convoluted and framework subordinate strategy, the aftereffect of which allows the source to adjust a way's rummaging utmost. As far as possible is described as the total number of foragers allowed to be enrolled and use the path later on. In more detail, every time another fabricated move is released for a particular way, a bit number of foragers is incorporated (enlisted) to the way's looking cutoff. This procedure ensures those ways which release more positive moves will end up with higher searching cutoff points and end up being more dynamic over the whole deal, than others which release negative moves or no moves by any methods.

\subsection{Adaptive Foraging}

The scouting methodology is seen as productive when one or more ack_scout is gotten by the source node. BIABRP concentrates on checking and continually surveying various ways. Right when a forager is gotten by the destination node, it passes on piggybacked data to the vehicle layer and changes over to an ack forager. Like the certified honeybees which put aside some time on the blossom to accumulate the dust or the nectar, the ack_forager stays at the destination node until some data package needs to do an inversion to the starting source. While doing a reversal home, it accumulates outstanding information from the nodes it visits and the associations between them. This grants it to screen the as a rule way's quality and have the ability to report the finding back to the source. The way is seen as a chain of associations between the center nodes, from the destination back to the source. While crossing it, the ack_forager is responsible for social occasion data that identify with the current state of its senders, and additionally, the framework ampleness of each widely appealing join.

The low's once-over level parameters used by the protocol, trailed by a brief illumination of their noteworthiness are given underneath.

1. The ack_forager's sign quality at the tolerant node in Watts. Being a bundle itself, when gotten it passes on sign quality. Fragile sign quality is an indication of long partition between the nodes and/or widely appealing preventions that impact the transmission.

2. The moving rate of the sender (speed) in $\mathrm{m} / \mathrm{s}$. A moving node can without a doubt go outside the transmission range and cause weak or broken associations. For the same reason, a node with a modified position is more reassuring than one that moves around persistently and at fast.

3. The sender's remaining imperativeness level in Joules. Nodes with sufficient remaining essentialness are less weak and better probability for future group transmissions.

4. The size of the Mac line of the sender in bits. The precise size of the line at the Mac layer of the sender is an indication of how involved the sender is similarly as development and framework stop up. 
5. The transmission delay between the sender and the beneficiary of an association in seconds. The use of time-stamps and synchronized timekeepers allows the time's estimation an ack_forager requires to complete a transmission from the sender to the recipient of an association.

Each one of these parameters can be isolated by getting to the fitting layers of the OSI model using cross-layering routines. The application layer can give both the moving rate and the remaining imperativeness resource. The physical layer can give the bundle's sign quality upon receipt. Knowing the Mac's compass layer's line and the allowed data rate as described by the 802.11 framework models of the remote connector, the coating deferral can be found. Finally, the transmission deferral is discovered by considering the time-stamps highly involved with sending and getting the group.

In order to be used effectively, the above parameters ought to be institutionalized, which is done by applying direct change. Notice that the moving pace and both line size and transmission deferral are unfavorably impacting the execution. A node's pace proportional to zero does not impact the transmission, as it doesn't alter the detachment between the source and the destination. This makes it perfect stood out from a node of high moving rate. Additionally, a node which has the most diminutive Mac bolster size is seen as free of blockage. Hence, banter institutionalization is used for these parameters. The piece of each parameter is basic yet they differentiate in the way they impact the execution and thusly the quality. This thought is in like manner met in bona fide honeybees' quality appraisal, where regardless of the way that as far as possible qualities are dark, it is tentatively found that they accept different parts and are joined together. Along these lines, to use them profitably a way to deal with express their relative criticalness ought to be found. In BIABRP's blueprint, a weighting structure using the machine learning method, Produced Neural Frameworks (ANN) is used consequently. This was refined by applying logged off regulated learning in perspective of data sets made by the remote framework. The readiness' goal was to find a course of action of weights that cause the yield from the neural framework to facilitate the honest to goodness target estimations of the data sets, as eagerly as would be reasonable. Full purposes of enthusiasm of the weight regard determination technique can be found in. The formula to find out the quality $\mathrm{q}$ of an association from node $\mathrm{j}$ to $\mathrm{k}$ as crossed by administrators $\mathrm{b}$ is showed up in the going with correlation:

$$
q_{j k}=\operatorname{sig}_{b}^{\prime} * w_{\text {sig }}+\operatorname{speed}_{j}^{\prime} * w_{\text {speed }}+\text { energy }_{j}^{\prime} * w_{\text {energy }}+q d_{j}^{\prime} * w_{q k}+t x d_{j k}^{\prime} * w_{t x d}---(1)
$$

where the prime numbers are the institutionalized estimations of the parameters (sig for signal's quality, et cetera.) and the $w_{s}^{\prime}$ are the fitting weights.

Taking after the trade as to the way of an association, another $\mathrm{q}$ is registered at every node passed by the ack_forager, and when it finally arrives home, the path's tendency from the destination d to the source $\mathrm{s}$ can be conveyed as:

$$
Q_{d s}=\sum_{n=1}^{m-1}\left(q_{N_{n+1} \rightarrow N_{n}}\right),\left[d=N_{m}, s=N_{1}\right]
$$

where $\mathrm{m}$ is the aggregate number of nodes in a numerically requested way and $N_{n+1} \rightarrow N_{n}$ is the pair of nodes with course towards the source node.

The outcome acquired by Eq. (2) is a number that can be utilized to speak to the present nature of the way, regarding the five low-level parameters. On the other hand, it speaks to stand out single flight. The system's way is such, that getting and utilizing the aftereffects of a solitary bundle transmission can be very deceptive. In BIABRP's structural engineering, results from a steady number of past flights are gathered, and in light of them, the source has the capacity research whether there has been a change or decay to the way execution after some time. Once there is adequate measure of information accessible, the last stride of the system is to apply relapse investigation and specifically, Pearson's connection coefficient. The thought of utilizing relapse examination is to get any solid positive or negative relationship between's the two variables, for this situation: time and the nature of a way $Q^{d s}$.. In such way any profitable changes can be measurably distinguished. Revised to fit BIABRP's configuration, the Pearson's $r$ is computed as

$$
r=\frac{\sum_{i=1}^{k}\left(t_{i}-\mu t\right)\left(Q_{i}^{d s}-\mu Q^{d s}\right)}{\sqrt{\sum_{i=1}^{k}\left(t_{i}-\mu t\right)^{2}} \sqrt{\sum_{i=1}^{k}\left(Q_{i}^{d s}-\mu Q^{d s}\right)^{2}}}
$$

here $t_{i}$ is the season of tolerating $\mathrm{Q}_{-}{ }^{\wedge} \wedge \mathrm{ds}, \mu \mathrm{t}$ the time's mean portion qualities and $\mathrm{k}$ the amount of flights accumulated.

As certifiable honeybees do in nature, their fake accomplices must gatherings a framework to recognize whether the way they are using stays exemplary. Besides, the standard of honeybee moving, when they have enough affirmation and a sensible appreciation of the way quality, they start the system of enlistment. In the protocol's setting, this is implied assembling enough data from past flights. If the relationship is an in number adverse, then the sign which is given to the enrolled individuals is moreover hostile, diminishing the way's looking point of confinement. If the association is an in number positive, as far as possible is extended. As far as possible for getting the strong connections are set precisely to - 0.8 and 0.8 . Regardless, dependent upon the 
utilization these edges can be changed, adjusting the affectability of both the checking and scrounging activities. Unmistakably, the closer to - 1 and 1 these breaking points get, the less tricky the protocol gets the opportunity.

\subsection{Selection of Ideal Paths}

Contingent upon the routing's conduct protocol that one might need to accomplish; diverse choice measurements can be connected. On their way back, foragers gather that data and check every way with a determination metric worth. Conventional metric qualities are identified with the quantity of bounces in a way, the transmission rate of its connections, the normal transmission check, the vitality cost, the remaining vitality, and so forth. Understanding the significance of this metric and its impact on the protocol's conduct is extremely noteworthy. For the exploratory correlation of this work, a metric identified with rate is utilized; the half's summation (round) transmission postpone and lining deferral for every middle of the road connection of the way, from the destination towards the source. This guarantees that the quickest way from the rundown is chosen. This sort of choice is done just at the source node. At the halfway nodes, the choice is finished by considering the bearing and the way recognizable proof number. At the destination node the determination is done in a first-in-first-out design. This methodology whitewashes the retransmission delays, as well as keeps the bundle's measure little (contrasted with source routing), permitting other helpful routing data to be obliged, if fundamental.

\subsection{Identification of Path Disappointments}

In spite of the consistent checking and assessment of the ways, where agents can distinguish changing so as to unsettle influences and avert loss of association naturally to another option, connections between the nodes can even now break rather surprisingly. A versatile routing protocol should have an instrument to distinguish when a connection has been broken and redesign its routing learning. Since BIABRP is intended to assess routing in view of "way" level rather than "connection" level, join breakage inside of a way is recognized when no foragers return back to the source node inside of a timeframe. The source node sets the way's scrounging ability to 0 and imprints the way as unacknowledged. The primary guarantees that no future foragers will be given the broken way's ID, though the last permits the way to end up accessible once more, if a forager in the long run returns. Besides, keeping in mind the end goal to dispose of extremely old unacknowledged ways, a clock is utilized for housekeeping. This straightforward instrument guarantees that control overhead stays low in light of the fact that no unique messages should be traded just to affirm nodes' presence. Regarding the halfway and the destination nodes, a clock pruning is additionally activated and unused routing data is evacuated.

\section{PERFORMANCE EVALUATION}

In this section, we evaluate BIABRP using simulations. There are no publicly available implementations of existing CRMANET or CRAHN routing protocols [G.Cheng et al.,2007, A.Sampath et al.,2008, G.M.Zhu et al.,2008, K.Chowdhury and I.Akyildiz.,2011, A.Cacciapuoti et al.,2012]. Also, many key implementation details are not clear enough for us to truthfully realize the functionalities they provide. The simulation code was written in C++. Initially, we tried the NS2 simulator and found that BIABRP has similar packet delivery ratio (PDR) to that of TIGHT in small-scale CRAHN. In terms of end-to-end latency, BIABRP only slightly outperforms TIGHT due to the small network scale. Since NS2 is known to have a poor performance in simulating large-scale CRAHN, we opted for $\mathrm{C}++$ simulations to see the performance of BIABRP in large-scale networks. The fidelity of $\mathrm{C}++$ simulations for BIABRP and TIGHT is still very high. Specifically, the routing of every packet in both BIABRP and TIGHT depends on the spontaneous network topology only. Therefore, we can simply evaluate the performance of BIABRP and TIGHT under node random walk mobility by sending packets over a number of randomly generated network topologies. We chose to compare TIGHT with GPSR which sends packets over the secondary channel only.

The default simulation settings are as follows: We simulate an area of 4,000 $\times 3,000 \mathrm{~m}^{2}$ with 6 PUs and 1,334 SUs. The transmission range of PUs and SUs is $250 \mathrm{~m}$. We run the simulation 8,000 times, and the PUs and SUs are uniformly distributed at random with a different random seed in each simulation run. We fix 30 pairs of source and destination PUs with random relative locations in each simulation run. BIABRP is used only when the source and destination SUs are both outside of any PU region; otherwise, TIGHT is used to send packets over the secondary channel only. Each source SU sends one packet of 512 bytes to its destination SU in each simulation run. Therefore, totally 8,000 packets are sent between each source destination SU pair, and each data point in subsequent figures represents the average for 240 thousand packets (unless stated otherwise). 


\section{RESULTS AND DISCUSSION}

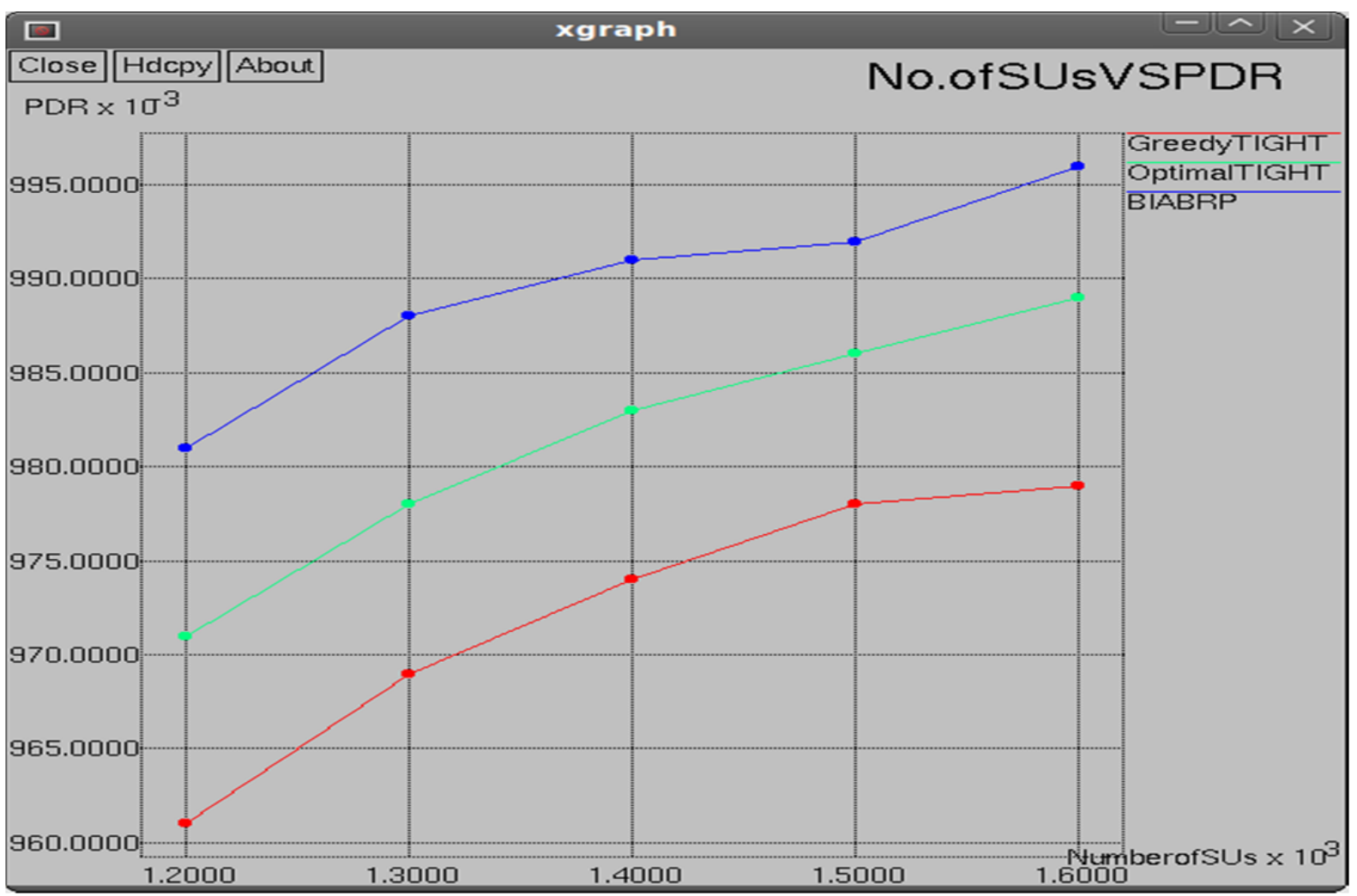

Figure 5.1: Impact of different numbers of SU's on packet delivery ratio.

Figure 5.1 shows the performance evaluation of the proposed BIABRP protocol in terms of number of secondary users versus packet delivery ratio. It is evident that the proposed BIABRP attains better packet delivery ratio. The numerical results are also presented in Table 5.1.

Table 5.1: Impact of different numbers of SU's on packet delivery ratio.

\begin{tabular}{|c|c|c|c|}
\hline No of SU's & Greedy TIGHT & Optimal TIGHT & BIABRP \\
& & & \\
\hline 1200 & 0.961 & 0.971 & 0.981 \\
\hline 1300 & 0.969 & 0.978 & 0.988 \\
\hline 1400 & 0.974 & 0.983 & 0.991 \\
\hline 1500 & 0.978 & 0.986 & 0.992 \\
\hline 1600 & 0.979 & 0.989 & 0.996 \\
\hline
\end{tabular}




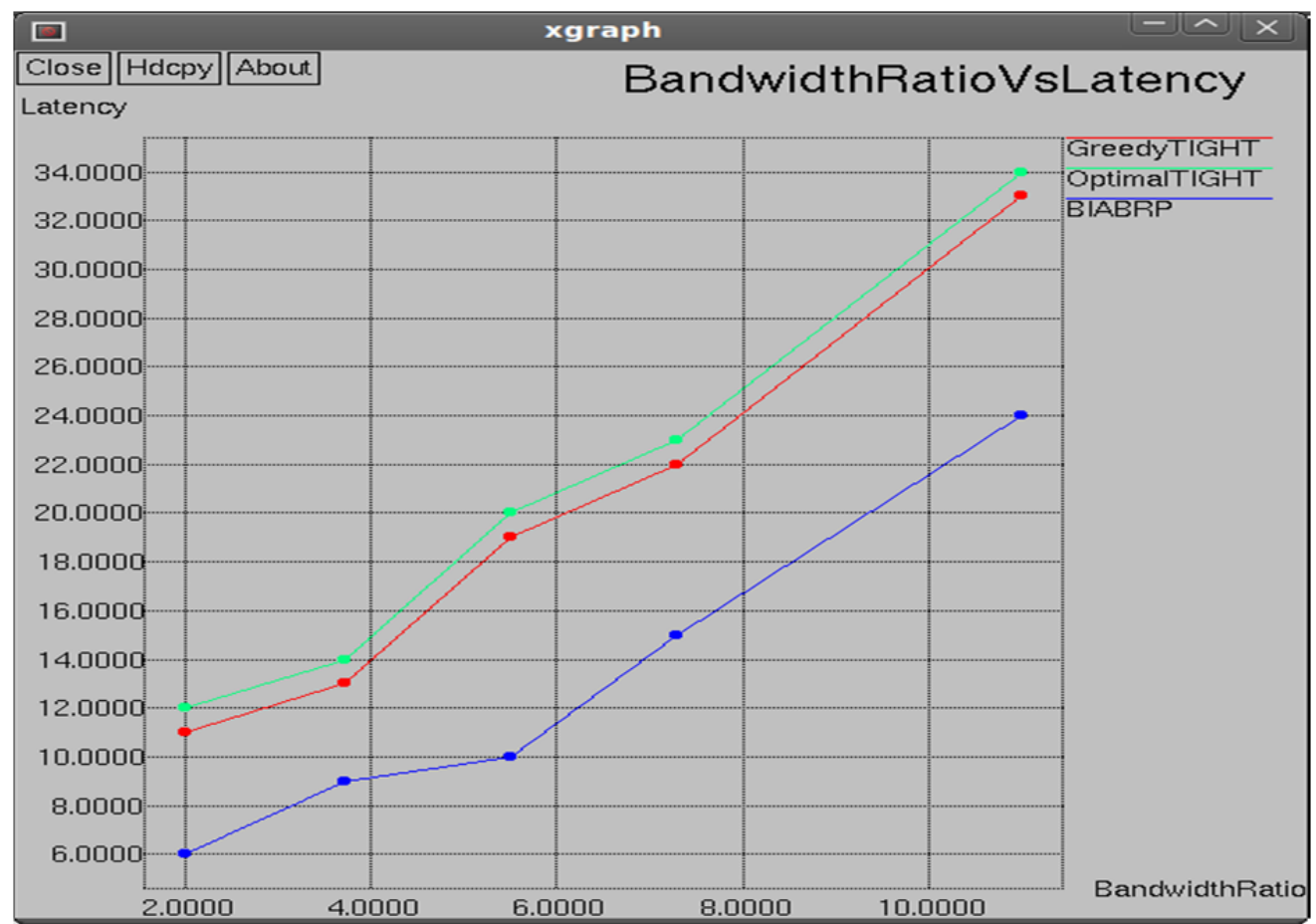

Figure 5.2: End-to-end latency

Figure 5.2 shows the performance evaluation of the proposed BIABRP protocol in terms of end-to-end delay. It is flagrant that the proposed BIABRP attains lesser end-to-end delay. The numerical results are also presented in Table 5.2.

Table 5.2: End-to-end latency

\begin{tabular}{|c|c|c|c|}
\hline Bandwidth Ratio & Optimal TIGHT & Greedy TIGHT & BIABRP \\
\hline 2 & 12 & 11 & 6 \\
\hline 3.7 & 14 & 13 & 9 \\
\hline 5.5 & 20 & 19 & 10 \\
\hline 7.3 & 23 & 22 & 15 \\
\hline 11 & 34 & 33 & 24 \\
\hline
\end{tabular}

\section{Conclusion}

This research work aims in design and development of bee inspired agent based routing protocol (BIABRP) for CRAHNs. Adaptive scouting, adaptive foraging, optimal path selection and detection of path removals are performed using honey bee inspired strategy. NS2 simulation tool is used to examine the performance of the proposed BIABRP. Appropriate performance metrics are chosen in order to conduct performance evaluation and the results prove that the proposed BIABRP outperforms in terms of packet delivery ratio, end-to-end latency and primary users activity.

\section{References:}

[1] A.Cacciapuoti, M.Caleffi, and L.Paura, "Reactive routing for mobile cognitive radio ad hoc networks," Ad Hoc Netw., vol. 10, no. 5, pp. 803-815, Jul. 2012.

[2] A.Sampath, L.Yang, L.Cao, H.Zheng, and B.Zhao, "High throughput spectrum-aware routing for cognitive radio networks," in Proc.CROWNCOM, Singapore, May 2008, pp. 1-6. 
[3] Chengetanai, Gibson; O'Reilly, Grant Blaise, "Review of swarm intelligence routing algorithms in wireless mobile ad hoc networks," in Intelligent Systems and Control (ISCO), 2015 IEEE 9th International Conference on , vol., no., pp.1-7, 9-10 Jan. 2015

[4] Desai, R.; Patil, B.P., "Cooperative reinforcement learning approach for routing in ad hoc networks," in Pervasive Computing (ICPC), 2015 International Conference on, vol., no., pp.1-5, 8-10 Jan. 2015

[5] G.Cheng, W.Liu, Y.Li, and W.Cheng, "Spectrum aware on-demand routing in cognitive radio networks," in Proc. IEEE DYSPAN, Dublin, Ireland, Apr. 2007, pp. 571-574.

[6] G.M.Zhu, I.Akyildiz, and G.S.Kuo, "STOD-RP: A spectrum-tree based on-emand routing protocol for multi-hop cognitive radio networks," in Proc. IEEE GLOBECOM, Nov. 2008, pp. 1-5.

[7] Janacik, P.; Orfanus, D.; Wilke, A., "A Survey of Ant Colony Optimization-Based Approaches to Routing in Computer Networks," in Intelligent Systems Modelling \& Simulation (ISMS), 2013 4th International Conference on , vol., no., pp.427-432, 29-31 Jan. 2013

[8] K.Chowdhury and I.Akyildiz, "CRP: A routing protocol for cognitive radio Ad Hoc networks," IEEE J. Sel. Areas Commun., vol. 29, no. 4, pp. 794-804, Apr. 2011.

[9] Karthikeyan, D.; Dharmalingam, M., "Ant based intelligent routing protocol for MANET," in Pattern Recognition, Informatics and Mobile Engineering (PRIME), 2013 International Conference on, vol., no., pp.11-16, 21-22 Feb. 2013

[10] Kiran, K.; Shivapriya, T.; Singh, A.A.; Begum, N.; Ramya, R.; Shenoy, P.D.; Venugopal, K.R.; Patnaik, L.M., "Performance analysis of bee-hive routing in multi-radio networks," in Advance Computing Conference (IACC), 2014 IEEE International, vol., no., pp.360364, 21-22 Feb. 2014

[11] Kuen-Han Li; Jenq-Shiou Leu; Hoek, J., "Ant-Based On-Demand Clustering Routing Protocol for Mobile Ad-Hoc Networks," in Innovative Mobile and Internet Services in Ubiquitous Computing (IMIS), 2013 Seventh International Conference on, vol., no., pp.354-359, 3-5 July 2013

[12] Prasad, B.M.G.; Srinivas, P.V.S., "SAMR: Swarm Adaptive Multipath Routing Topology for Load Balancing and Congestion Endurance in Mobile Ad Hoc Networks," in Advanced Computing and Communication Technologies (ACCT), 2013 Third International Conference on, vol., no., pp.276-282, 6-7 April 2013

[13] Sharma, P.; Kumar, R., "Enhanced swarm intelligence-based scheduler to improve QoS for MANETs," in Confluence The Next Generation Information Technology Summit (Confluence), 2014 5th International Conference -, vol., no., pp.340-346, 25-26 Sept. 2014

[14] Thangadurai, K.; Anchugam, C.V., "Fuzzy Cost Based Multipath Routing Protocol for MANETS," in Computing and Communication Technologies (WCCCT), 2014 World Congress on, vol., no., pp.286-290, Feb. 27 2014-March 12014

[15] Zafar, S.; Soni, M.K., "Trust based QOS protocol(TBQP) using meta-heuristic genetic algorithm for optimizing and securing MANET," in Optimization, Reliabilty, and Information Technology (ICROIT), 2014 International Conference on, vol., no., pp.173177, 6-8 Feb. 2014

[16] RameshPalanisamy, V. Mathivanan “The Wireless Security Survey of India” Volume 5, Issue12, December 2015 ISSN: 2277 128X International Journal of Advanced Research in Computer Science and Software Engineering

[17] RameshPalanisamy,V.Mathivanan"Performane Evaluation Of Sacbrp Based On PDR"International Journal of Computational Intelligence Research ISSN 0973-1873 Volume 12, Number 1 (2016), pp. 69-81 @ Research India Publications.

[18] RameshPalanisamy, V. Mathivanan "Efficient Zone Based Routing Protocol (EZBRP)" International Journal of Computer Science and Information Security (IJCSIS), Vol. 14, No. 5, May 2016.

[19] RameshPalanisamy,V.Mathivanan"Performance Evaluation of SACBRP Based on End to End Latency" International Journal of Computer Trends and Technology (IJCTT) - Volume 35 Number 2- May 2016 ISSN: 2231-2803 Page 96.

\section{AUTHOR PROFILE}

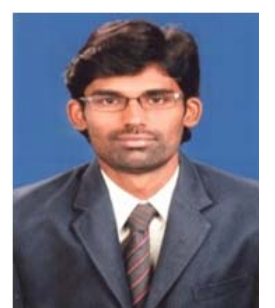

Ramesh Palanisamy obtained his Bachelor's degree in Electronics from Barathiar University Coimbatore, India. Then he obtained his Master's degree in Computer Communications, from Barathiar University Coimbatore, India. Currently doing Ph.D. in Computer Science and Engineering at AMET University, Chennai. His Reliable Data Transmission Over Cognitive Radio Ad Hoc Networks specialization includes Computer Networks.Technical Qualifications CCNA-(CiscoCertified Network Associate).NSP-(Network Support Professional).HNA- (Hardware Networking Administrator).CCSI - (Cisco Certified System Instructor).

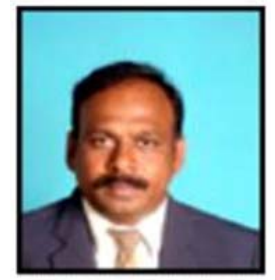

Dr. V.Mathivanan obtained his Bachelor's degree in Computer Science Bharathidasan University, Trichy. Then he obtained his Master's degree in Computer Science Bharathidasan University, Trichy and again he obtained his Master of Engineering in Computer Science and Engineering Anna University, Chennai. Master of Philosophy in Computer Science from Alagappa University, Karaikudi. Ph.D. degree in Computer Science and Engineering from Alagappa University, Karaikudi. Technical Qualifications C.C.A.ICisco Certified Academic Instructor,C.C.N.A-Cisco Certified Network Associates,Sun Solaris - Skill Drill Certification,IBM DB2 - IBM Database 2 Certified Professional,IBM Websphere Certified Developer,I.C.D.L- International Computer Driving License. He has published 20 papers in National and International Journals and 12 Papers Presented in Conferences. 